\title{
EFFECT OF INTERSECTION LIGHTING DESIGN ON DRIVERS' PERCEIVED VISIBILITY AND GLARE
}

\section{Rajaram Bhagavathula, Corresponding Author}

Virginia Tech Transportation Institute

3500 Transportation Research Plaza, Blacksburg, VA 24061

Phone: (540) 231-5209; Fax: (540) 231-1555; Email: Rbhagavathula@vtti.vt.edu

\section{Ronald Gibbons}

Virginia Tech Transportation Institute 3500 Transportation Research Plaza, Blacksburg, VA 24061

Phone: (540) 231-1581; Fax: (540) 231-1555; Email: Rgibbons@vtti.vt.edu

\section{Maury A. Nussbaum}

Department of Industrial \& Systems Engineering, Virginia Tech 521 Whittemore Hall (MC 0118), 1185 Perry Street, Blacksburg, VA 24061

Phone: (540) 231-6053; Fax: (540) 231-3322; Email: Nussbaum@vt.edu

Word count: 6,936 words text +2 table x 250 words (each) $=7,436$ words

29 Submission Date: November 15, 2018. 


\section{ABSTRACT}

A systems level approach to intersection lighting design has shown that illuminating the intersection box increases drivers' nighttime visual performance. However, for an intersection lighting design to be effective and accepted, it should not only maximize visual performance but also enhance perceived visibility and minimize glare. The goals of this study were to assess the effects of different intersection lighting designs on these two outcomes. Visibility was assessed with respect to a pedestrian, several targets, and an intersection. Perceptions of visibility and glare were measured using Likert scales, with participants exposed to multiple lighting designs on a realistic intersection. Twenty-four participants completed the study, with an equal number of younger (18-35 years) and older (65+) drivers. The lighting design that illuminated the intersection box had the highest levels of perceived target and intersection visibility and the lowest ratings of glare. For the same lighting configuration, a strong positive correlation was also found between perceived target visibility and previous results on target detection distances. In this configuration, perceived visibility plateaued between 7 and 10 lux of mean intersection illuminance. Increased levels of perceived visibility in different conditions were likely a result of size and contrast differences, and the distribution of the luminaires used. These results suggest that illuminating the intersection box has multiple benefits, in that it not only increases visual performance but also increases perceived visibility and reduces glare.

Keywords: Lighting, Intersections, Perceived Visibility, Perceived Glare, Night, Drivers, Pedestrians 
INTRODUCTION

Night crashes at intersections pose a major safety concern in the United States, as they account for about $40 \%$ of the total crashes at intersections (1). Installing lighting at intersections has been used as a successful countermeasure against night crashes. For example, lighting an intersection has reduced nightto-day crash ratios and rates by 13 to $45 \%$, respectively (2-6), and an increase in light levels has been associated with respective decreases in night-to-day crash ratios and rates of 7\% (7) and 9\% (8). Existing recommendations and guidelines for the design of intersection lighting have focused solely on lighting levels, and stemmed from research relating lighting to night crashes at intersections. This has ignored the role of human vision in intersection lighting design, as well as the interactive effect of vehicle headlamps and overhead lighting. Furthermore, existing standards (Illumination Engineering Society of North America and the Commission Internationale de l'Éclairage) prescribe minimum lighting levels to be maintained within the intersection box (area enclosed within the stop bars at the intersection), which does not account for the multiple pedestrian-vehicle conflict points at intersections.

A new systems level approach to intersection lighting design was introduced by Bhagavathula, Gibbons and Nussbaum (9). (Note that we use the term "intersection lighting design" to refer to both the part of the intersection illuminated and the recommended light level). In the noted paper, three intersection lighting designs were evaluated (Lighted Approach, Lighted Box, and Lighted Approach and Box as shown in FIGURE 1). This evaluation was done on the basis of drivers' nighttime visual performance, using objective measures of detection distances for targets located at the entrances, exits, and middle of pedestrian crosswalks at intersections. Results of the study indicated that the design that illuminated the intersection box offered better visual performance (longer detection distances as shown in FIGURE 2) and had fewer number of missed target detections. However, for an intersection lighting design to be effective and accepted it should not only increase a driver's visual performance but also increase perceived visibility and reduce glare. Pedestrian visibility is also important, since, as noted, existing intersection lighting designs do not account for pedestrian-vehicle conflict locations. Finally, it is important to know if intersection lighting configurations that support enhanced detection (longer target detection distances) are also perceived as having high visibility and low glare.

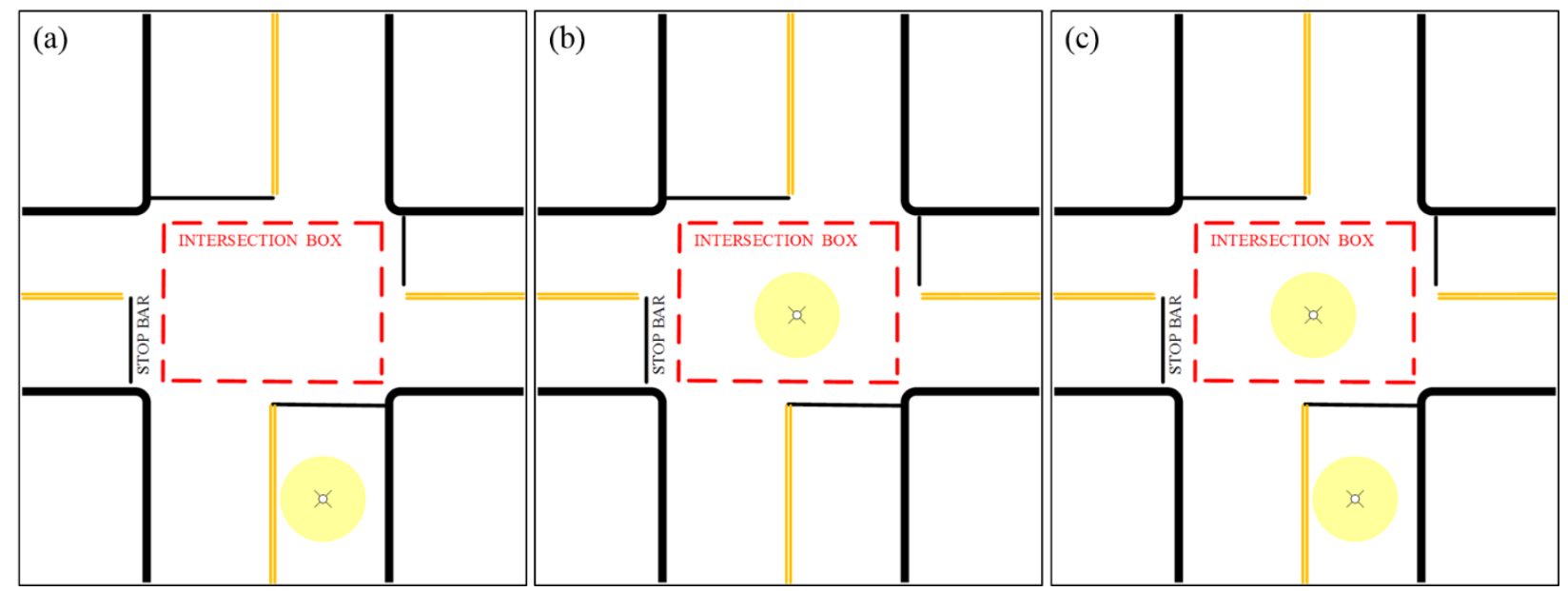

FIGURE 1 Illustrations of the three intersection lighting configurations: (a) Intersection approach is illuminated, (b) Intersection box is illuminated. (c) Both the box and approach are illuminated. The distribution of light (denoted by the yellow color) is not photometrically accurate and is only included to represent the light emitted by the luminaire for illustrative purposes. 


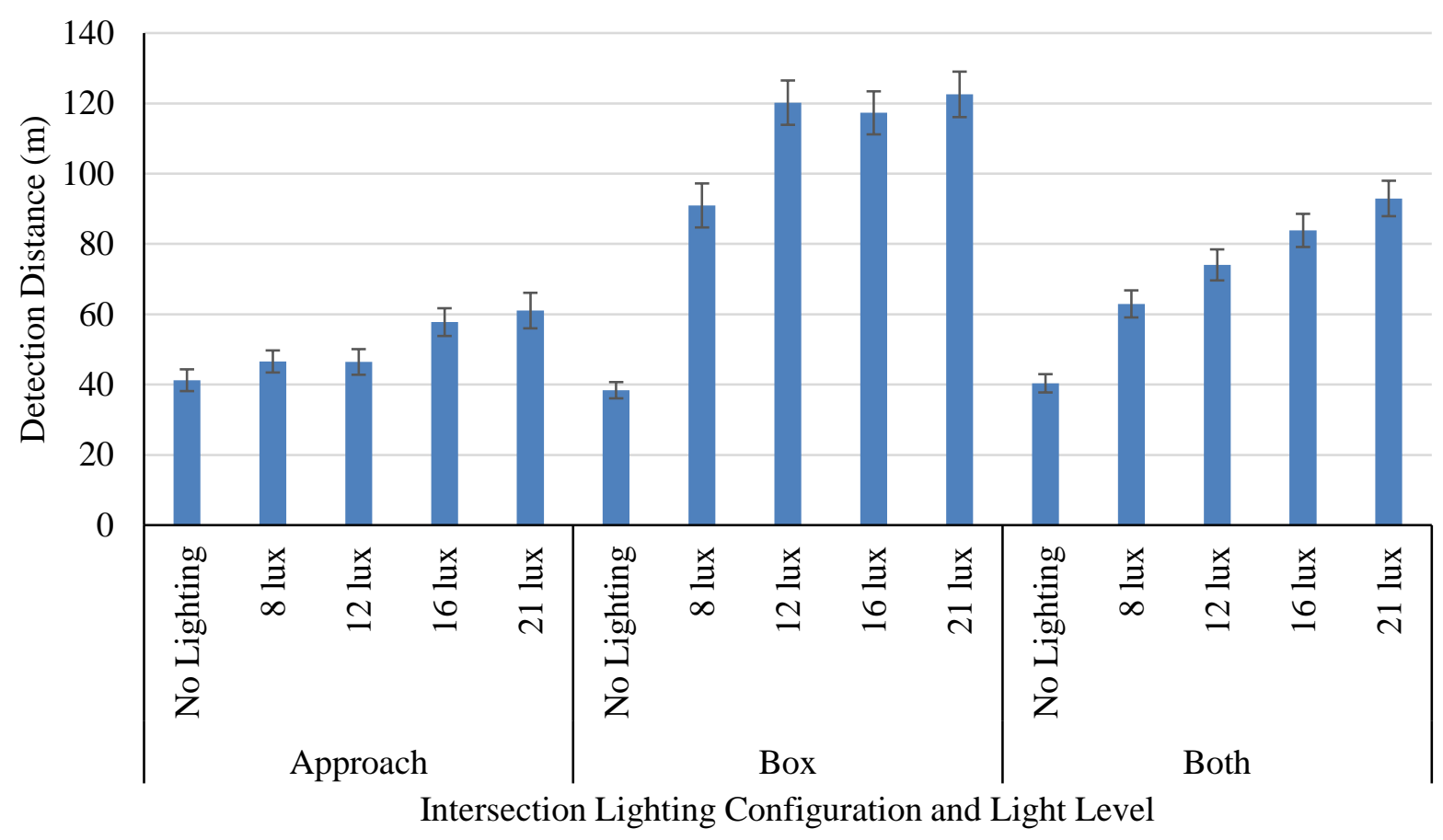

\section{FIGURE 2 Effect of intersection lighting configuration and light level on detection distance of drivers as reported in Bhagavathula, Gibbons and Nussbaum (9). Values reported are means of detection distances of standard visibility targets and errors bars represent standard errors.}

Earlier work has shown that perceived visibility is associated with nighttime driver visual performance. For example, Gallagher, Koth and Freedman (10) found that a visibility metric based on subjective ratings was a strong predictor of driver visual performance, while Janoff, Kroth, McCunney, Freedman, Duerk and Berkovitz (11) showed that subjective ratings of visibility can serve as a predictor of nighttime crashes. Janoff (12) also studied the relation between subjective ratings of visibility and a Visibility Index (VI). The VI is a measure of visibility and is dependent on contrast, relative contrast sensitivity, and disability glare. VI was found to be highly correlated with subjective ratings of visibility, and both the size of the object involved and the contrast were reported to affect the relationship between subjective ratings of visibility and VI. An increase in lighting level was also found to be associated with an increase in subjective ratings of visibility. Increases in lighting level have also been associated with increases in perceived ratings of glare (13-16).

However, only one existing report, to our knowledge, has examined the effect of intersection lighting design on subjective ratings of visibility. In this report (17), subjective ratings of visibility were obtained from drivers who were exposed to three different intersection lighting layouts (or configurations), each with three levels of illumination (5, 10 and 15 lux). The three intersection layouts were based on the part of the intersection that was illuminated, and used the following three configurations: approach, corner (or box), and both approach and corner. Drivers rated five statements: “visibility”, “danger to pedestrian”, “ease of driving”, "brightness” and "safety” on a Likert-type scale (1 to 5), and a mean rating > 3 (or "neutral" anchor the Likert-type scale) was used as indicating effectiveness of an intersection lighting design. In the noted study, increases in light levels resulted in higher subjective ratings of visibility. With light levels $>10$ lux, mean ratings of pedestrian visibility were higher than 3 on the Likert-type scale in all three layouts. Minoshima, Oka, Ikehara and Inukai (17) also found that ratings (all statements including pedestrian visibility) depended on the light level. At the 15 lux light level, the lighting configuration that illuminated the approach and corner was rated highest, while at the 10 and 5 lux light levels the configuration that illuminated the approach was rated the highest. 
The authors concluded that the approach lighting layout should be used to maintain a mean roadway surface luminance of 10 lux. However, if a higher level of mean roadway illuminance is needed, then both approach and corner illumination should be used. This study, though, did not measure perceived glare. Furthermore, only mean ratings were presented, with no statistical analyses reported regarding differences between lighting configurations or light levels. The specific age ranges of participants were also not described, other than that an unbalanced sample of "elder" $(n=5)$ and "non-elder" $(n=15)$ individuals were included. Thus, there is need for additional research that considers the perception of glare and that more formally quantifies differences in perceived visibility with different intersection lighting designs.

The current study assessed the effects of different intersection lighting configurations and light levels on perceived visibility and glare. We hypothesized that: (1) perceived visibility and glare will differ between the three lighting configurations and between ratings of pedestrians and target areas, similar to results reported by Janoff (12); and (2) increasing light level will result in higher perceived visibility, as reported by Janoff (12) and Minoshima, Oka, Ikehara and Inukai (17), and also result in higher perceived glare, as supported by existing research in this area (13-16). Results from this study were intended to supplement earlier results regarding visual performance (9), and to help determine whether intersection lighting designs that result in better visual performance also lead to improved visibility and lower glare.

\section{METHODS}

This study was performed in conjunction with the work reported earlier (9) and used many of the same methods. Twenty-four participants completed the study, and were recruited to form two age groups younger $(\mathrm{M}=30.8$ years, $\mathrm{SD}=2.7)$ and older $(\mathrm{M}=68.2$ years, $\mathrm{SD}=1.6)$ - with same number of male and female participants in each group. The selected age groups were intended to account for a wide range of driving experiences and visual capabilities. All participants had a valid US driver's license and a minimum visual acuity of 6/12 (20/40). Participants' visual acuity was assessed using a basic visual acuity test, administered using an Early Treatment Diabetic Retinopathy Study (ETDRS) chart with an illuminator cabinet. Experimental sessions were conducted on three separate nights after an initial screening session. Participants were compensated \$30 per hour for their participation. All experimental protocols were approved by Virginia Tech's Institutional Review Board, and all participants provided written informed consent prior to any data collection.

\section{Experimental Design}

The effects of intersection lighting configuration and light level on participants' perceptions of pedestrian visibility, target visibility, intersection visibility, and glare were evaluated using a repeated-measures factorial design. Participants were exposed to both fixed targets and a simulated pedestrian, under multiple lighting configurations and light levels at a realistic roadway intersection. This intersection was on the Virginia Smart Road, located at the Virginia Tech Transportation Institute. The Smart Road is a controlled access roadway research facility built to United States Federal Highway Administration specifications, and the intersection is fully functional, two-lane, four-way, and signalized (FIGURE 3a). Participants encountered each of three lighting configurations, in separate data collection sessions on different nights, and five light levels within each lighting configuration. Data collection occurred only on clear nights (no rain, snow or fog) and after civil twilight. The order of presentation of lighting configurations and light levels was counterbalanced to minimize order effects. The locations at which the targets were presented was also counterbalanced, with catch trials included (no target presentation). 


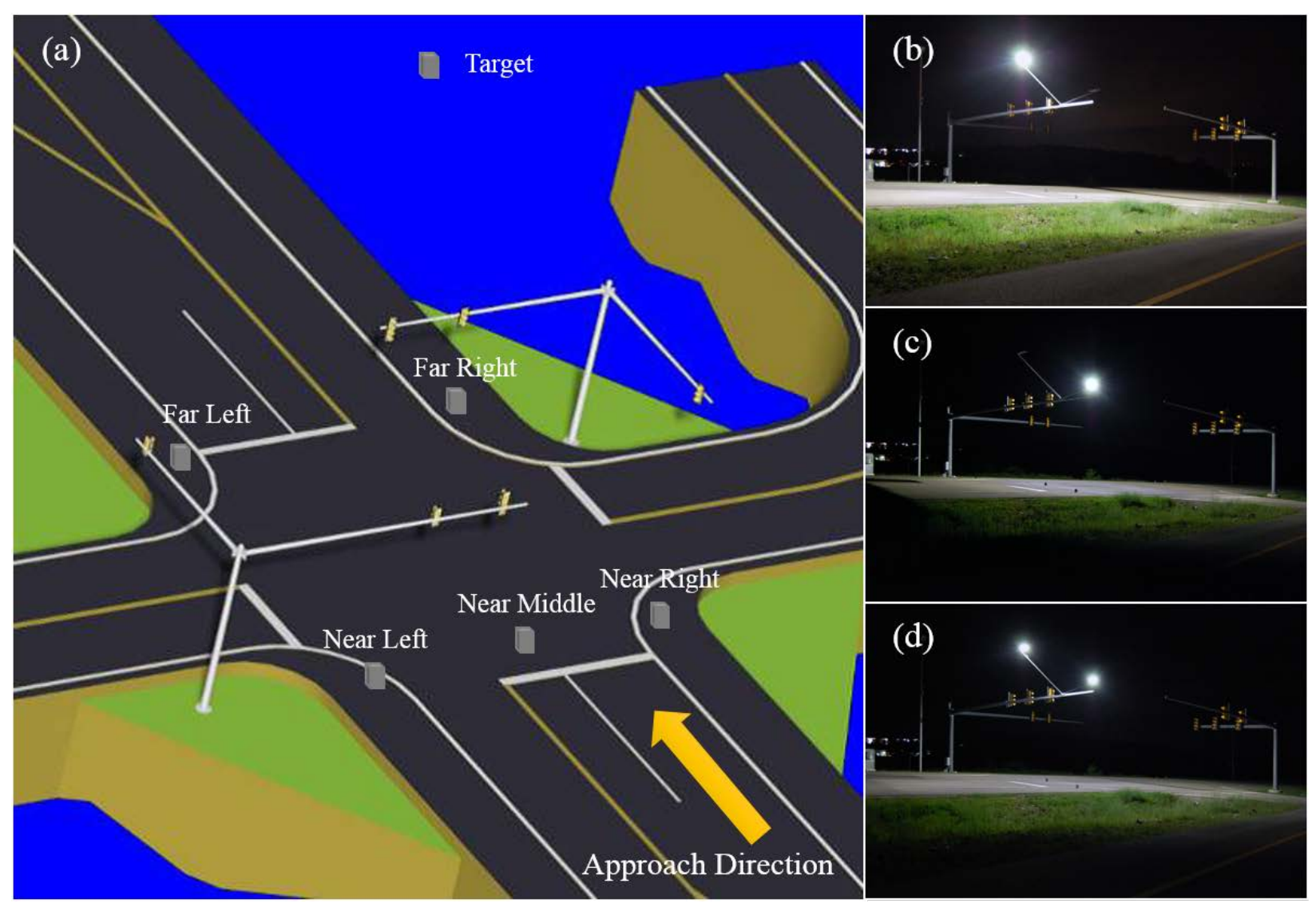

FIGURE 3 Diagram of the intersection on the test track with target locations indicated (a). The intersection is equipped with signal lights and lane markings. The intersection could also be illuminated by three separate lighting configurations, which illuminated the intersection approach (b), the intersection box (c), and both the intersection approach and box (d).

\section{Targets}

Targets were used to evaluate roadway visibility (by measuring detection distance in the visual performance experiment); these were of grey color and $0.18 \times 0.18 \mathrm{~m}$ in size, similar to ones used in earlier work (18-25). Targets were located 0.3 meters outside the right shoulder of the road. Five target locations were used in the study (see FIGURE 3a), and were located at the pedestrian-vehicle conflict locations at the entrances, exits, and the middle of the crosswalks located at the intersections.

\section{Pedestrian}

A simulated pedestrian was achieved using a member of the experimental team (stature $=1.77 \mathrm{~m}$ ), and was clothed in gray medical scrubs (FIGURE 4). A gray color was chosen because it is a neutral color and would be rendered similarly under different lighting configurations and light levels. 


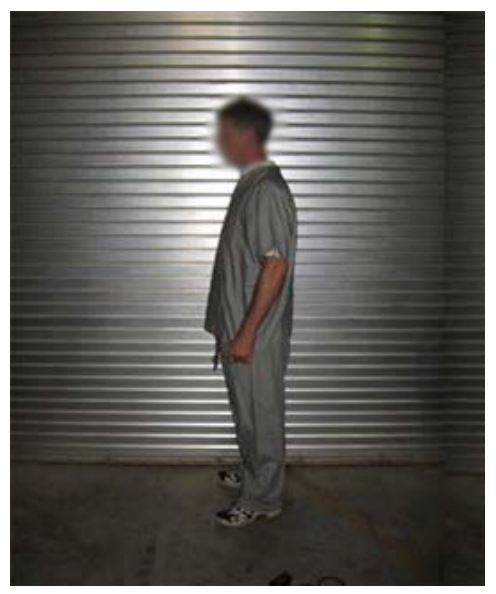

\section{FIGURE 4 Simulated pedestrian, wearing gray medical scrubs.}

\section{Intersection Lighting Configurations}

Three intersection lighting configurations were used. In the first, or Approach lighting, the approach to the intersection was illuminated (FIGURE 3b). In the second configuration (Box), the intersection box was illuminated (FIGURE 3c), while the third configuration (Approach and Box or Both) illuminated both the approach and box (FIGURE 3d). These lighting configurations also rendered an object (Target or Pedestrian) located at the near right, near middle, and near left target locations in different contrasts for an approaching driver. The Approach configuration rendered objects in these locations in positive contrast, whereas the Box configuration rendered them in negative contrast. Contrasts of the near right, near middle, and near left targets in the Both lighting configuration depended on the light level. The remaining two target locations (far right and far left) always appeared in positive contrast, as their facing side was always brighter than the background by the virtue of their location with respect to the lighting configurations.

\section{Intersection Light levels}

Illumination was provided by two 4000 Kelvin light-emitting diode (LED) luminaires that were mounted at a height of $8.5 \mathrm{~m}$. Both luminaires were purchased from the same manufacturer (CREE) and from the same product catergory (LEDway ${ }^{\circledR}$ ), and differed only in the type of light distribution; we thus assumed they had nearly identical spectral power distributions. One luminaire was used to illuminate the intersection approach and the other illuminated the intersection box. The former luminaire had a Type II medium distribution, while the one illuminating the intersection box had a Type V medium distribution. Each lighting configuration was illuminated to five light levels, specifically 0 (no lighting), 8, 12, 16, and 21 lux. These levels corresponded to the horizontal light levels at the pedestrian/near right target location on the road surface and were selected so that they could be could be obtained in all three lighting configurations. The vertical illuminance for the pedestrian location and the mean horizontal illuminance of the intersection (mean intersection illuminance) are shown in TABLE 1 for each lighting configuration and light level. Mean horizontal illuminance was the average horizontal roadway surface illuminance of the area enclosed by the stop-bars at the intersection. Light levels used were based on the IES RP-8 recommended average light levels for pedestrian volumes areas (low, medium and high) at rural, suburban, and urban areas (25).

TABLE 1 Vertical illuminance at the pedestrian location and mean horizontal intersection illuminance used in the study for each lighting configuration and illuminance 


\begin{tabular}{|c|c|c|c|}
\hline $\begin{array}{l}\text { Intersection } \\
\text { Lighting } \\
\text { Configuration }\end{array}$ & $\begin{array}{l}\text { Horizontal } \\
\text { Illuminance } \\
\text { (lux) }\end{array}$ & $\begin{array}{l}\text { Vertical Illuminance on } \\
\text { Pedestrian (Measured at } \\
1.5 \mathrm{~m} \text { above the road } \\
\text { surface) (lux) }\end{array}$ & $\begin{array}{l}\text { Mean } \\
\text { Intersection } \\
\text { Illuminance } \\
\text { (lux) }\end{array}$ \\
\hline \multirow{4}{*}{ Approach } & 8 & 5 & 5 \\
\hline & 12 & 7 & 7 \\
\hline & 16 & 10 & 9 \\
\hline & 21 & 13 & 12 \\
\hline \multirow{4}{*}{ Box } & 8 & 1 & 7 \\
\hline & 12 & 1 & 10 \\
\hline & 16 & 1 & 13 \\
\hline & 21 & 2 & 18 \\
\hline \multirow{4}{*}{ Both } & 8 & 9 & 5 \\
\hline & 12 & 13 & 8 \\
\hline & 16 & 17 & 11 \\
\hline & 21 & 22 & 13 \\
\hline
\end{tabular}

1

\section{Experimental Procedure}

Experimental procedures were very similar to those used in the noted previous paper (9). Two participants were scheduled for each experimental session, and upon arrival they initialled the informed consent form and were provided with an overview of the experimental session by an experimenter in one of the vehicles used (Ford Explorers, model years 1999 and 2000). This overview included a presentation of images of the targets and pedestrian they would be seeing on the road and an explanation of how to provide ratings using questionnaires (described below). Participants were shown the questionnaire and were given an explanation about the meaning, purpose, and significance of each statement. Low-beam headlamps were used during the study. The vehicles' headlamps were Hella $90 \mathrm{~mm}$ Bi-Xenon projector lamps with a single 1-F capacitor-stabilized headlamp input voltage on each vehicle. These headlamps were retrofitted along with a voltage stabilizer so that the headlamps' intensities were not affected by speed of vehicles. These retrofitted headlamps also allowed the headlamps to be at the same height from the ground across both the experimental vehicles. Before every experimental session, vehicle headlamps were aimed and the windshields were wiped clean.

Participants performed two separate tasks in each of the three data-collection sessions (see FIGURE 5). The first was a target detection task, in which participants detected targets and their detection distances were measured (9). The second task was the questionnaire rating, in which participants rated the intersection lighting configuration and the light level with a simulated pedestrian standing at the intersection.

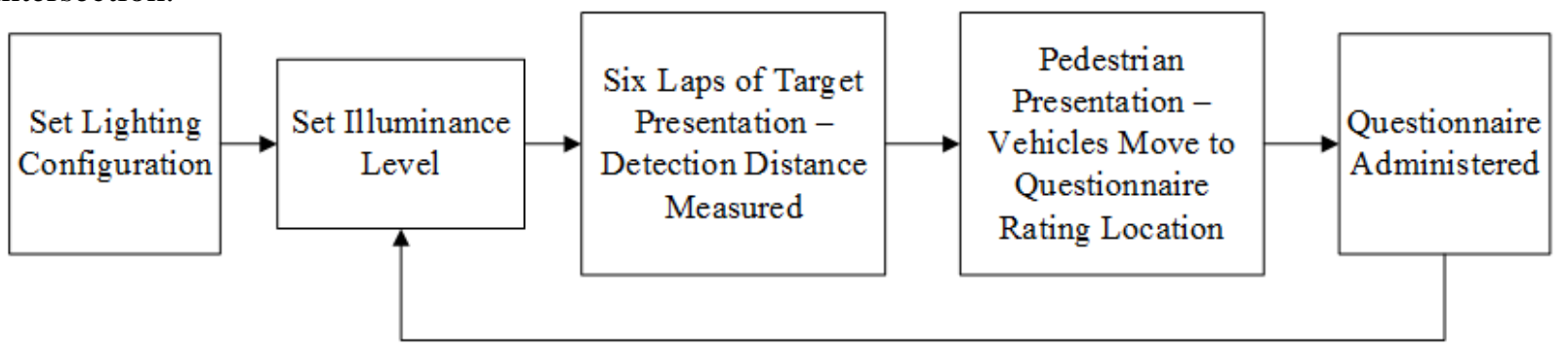


Participants approached the intersection six times for each light level and lighting configuration, during which they performed the noted target detection task. The speed limit for the detection task was $56 \mathrm{~km} / \mathrm{h}$ (35 mi/h), which was selected as it was the upper end of the speed limit in urban/residential areas in the Commonwealth of Virginia. Before starting the target detection task, participants were asked to remember how that particular combination of lighting configuration and light level affected their perception of target visibility (the questionnaire contained specific statements about target visibility).

Questionnaire ratings were solicited after participants completed all target detections for a given light level. Both participants were first asked to drive to the location in the intersection approach marked by a cone (see FIGURE 6). The two experimental vehicles were parked next to each other, with the second vehicle's headlamps turned off. This was done for efficiency, allowing both participants, in the two vehicles, to rate the questionnaires in parallel. The cone (FIGURE 6) was located $76.2 \mathrm{~m}$ from the location of the simulated pedestrian, who stood in the right shoulder at the entrance to the nearest crosswalk and always faced the roadway (FIGURE 6). This specific distance was used, as it is the stopping sight distance for the "design speed" of $56 \mathrm{~km} / \mathrm{h}(35 \mathrm{mi} / \mathrm{h})(26)$, or the distance along the roadway required for a vehicle travelling at the design speed to come to a complete stop.

Once both the experimental vehicles were parked at the cone facing the intersection with the pedestrian, they were administered the questionnaire by the in-vehicle experimenter. Participants rated their level of agreement with several statements, using a custom questionnaire developed for this study (Figure 7). The questionnaire was piloted tested for consistency of responses prior to use in the study. There were a total of 10 statements, assessing four areas - Pedestrian Visibility, Target Visibility, Intersection Visibility, and Glare - with responses obtained using Likert Scales. Pedestrian Visibility (statements 1 and 7) and Target Visibility (statements 2 and 6) were each assessed using two statements, while Intersection Visibility (statements 4, 9 and 10) and Glare (statements 3, 5 and 8) were assessed with three statements each. Established glare rating scales such as the deBoer discomfort glare rating scale was not used because it was reported to be a bad predictor of night driving performance (16). After completing the questionnaire, participants travelled back to the starting point and prepared for the next trial, at the next light level. The same protocol was repeated in the second and third data collection sessions, using the other two lighting configurations. 


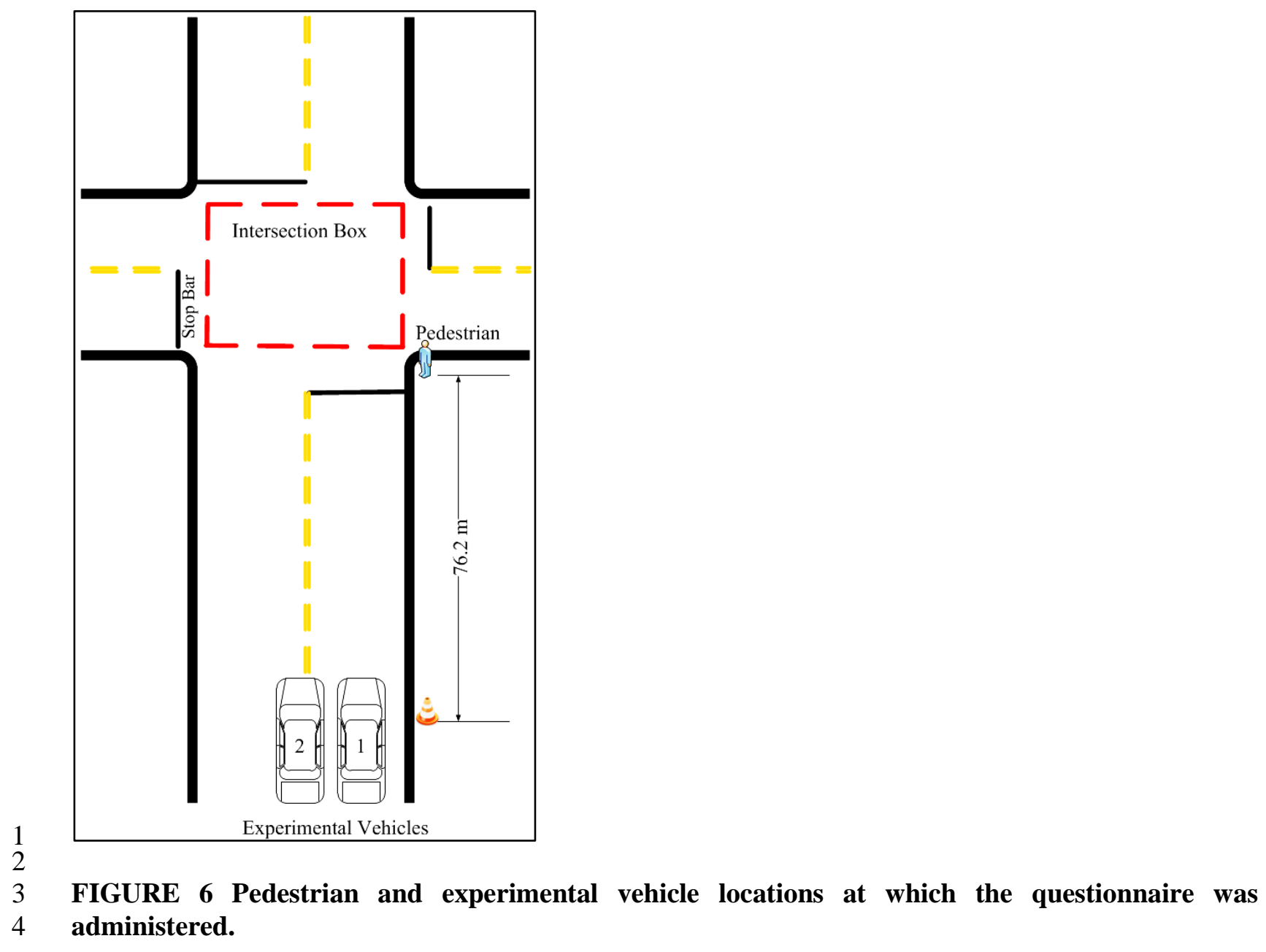


Please indicate how much you agree or disagree with each of the following statements with respect to the current lighting level (please the check the appropriate box)

\begin{tabular}{|c|c|c|c|c|c|c|}
\hline \multirow{2}{*}{ No } & \multirow{2}{*}{ Statement } & $\begin{array}{l}\text { Strongly } \\
\text { Disagree }\end{array}$ & Disagree & Neutral & Agree & $\begin{array}{c}\text { Strongly } \\
\text { Agree }\end{array}$ \\
\hline & & 1 & 2 & 3 & 4 & 5 \\
\hline 1 & $\begin{array}{l}\text { The pedestrian is clearly } \\
\text { visible }\end{array}$ & & & & & \\
\hline 2 & $\begin{array}{l}\text { All targets were seen from a } \\
\text { safe distance }\end{array}$ & & & & & \\
\hline 3 & $\begin{array}{l}\text { I experienced glare when } \\
\text { approaching the intersection }\end{array}$ & & & & & \\
\hline 4 & $\begin{array}{l}\text { It was easy to drive through } \\
\text { the intersection }\end{array}$ & & & & & \\
\hline 5 & $\begin{array}{l}\text { Glare from the overhead } \\
\text { lighting is affecting my ability } \\
\text { to see the pedestrian }\end{array}$ & & & & & \\
\hline 6 & $\begin{array}{l}\text { All targets were clearly } \\
\text { visible }\end{array}$ & & & & & \\
\hline 7 & I can easily see the pedestrian & & & & & \\
\hline 8 & $\begin{array}{l}\text { Glare from overhead lighting } \\
\text { is affecting my ability to } \\
\text { detect targets }\end{array}$ & & & & & \\
\hline 9 & $\begin{array}{l}\text { All the roads leading to the } \\
\text { intersection are clearly visible }\end{array}$ & & & & & \\
\hline 10 & Intersection is clearly visible & & & & & \\
\hline
\end{tabular}

Figure 7 Likert scale questionnaire used for subjective ratings of pedestrian visibility, target visibility, intersection visibility, and glare.

Analyses

Inter-item reliability of the questionnaire responses was assessed using standardized Cronbach's alpha values for each of four assessment areas. Additional analyses were only conducted when Cronbach's alpha was $>0.7$, indicating that there was a high level inter-item reliability in the questionnaire statements for a particular assessment area (27). Standardized Cronbach's alpha values were as follows: Pedestrian Visibility $=0.95$, Target Visibility $=0.94$, Intersection Visibility $=0.85$, and Glare $=0.78$. Given this high level of reliability, composite Likert scores were calculated for each assessment area, as mean ratings across multiple statements in each assessment area. These composite scores were used as dependent measures.

Separate linear mixed models (LMM) were used to assess the effects of lighting configuration and light levels on composite scores in each of the four assessment areas (pedestrian visibility, target visibility, intersection visibility, and glare). Age group was used as a blocking variable. Preliminary analyses indicated that no main or interactive effects involving gender were significant. Thus, gender was 
not included in the final models. For all statistical tests, the significance level was established at $p<0.05$. Where relevant, post hoc pairwise comparisons were performed using Tukey's honestly significant difference (HSD) for main effects, and simple effects testing was used to examine significant interaction effects. As in the approach used by Minoshima et al. (17), a particular lighting configuration and light level was considered effective only when the mean visibility ratings (Pedestrian, Target, and Intersection) were $>3$ (i.e., “Agree” or "Strongly Agree”) and mean Glare ratings were < 3 (i.e., "Disagree” or "Strongly Disagree"). The plateau in the ratings with increasing illuminance was determined based on observed pattern of effects of illuminance on ratings for the intersection lighting configurations. A plateau was considered to occur when an increase in light level did not result in a corresponding statistically significant increase in ratings in post hoc pairwise comparisons. Additionally, Pearson product-moment correlation coefficients were determined separately in each of the three lighting configurations, to assess the association between target detection distance (from the visual performance experiment, as reported in (9)) and the composite score of perceived target visibility.

\section{RESULTS}

LMM results of Likert-scale composite scores of pedestrian visibility, target visibility, intersection visibility, and glare are summarized in TABLE 2. Both lighting configuration and light level had significant main effects on all scores, excepting the effect of light level on glare, for which there was a significant interactive effect. For target visibility and intersection visibility, the interaction between lighting configuration and light level approached significance. Results for each of these assessment areas are presented in more detail in the following sections.

TABLE 2 Significant statistical results from linear mixed model analysis of the effects of age, lighting configuration, and light level on composite scores of pedestrian visibility, target visibility, intersection visibility, and glare

\begin{tabular}{|c|c|c|}
\hline Assessment Area & Effect & Statistical Results \\
\hline \multirow{3}{*}{ Pedestrian Visibility } & Lighting Configuration (LC) & $F(2,115)=5.63, p=0.005$ \\
\hline & Light Level (LL) & $\mathrm{F}(4,220)=82.07, \mathrm{p}<0.001$ \\
\hline & Age $x$ LL & $F(4,220)=2.43, p=0.048$ \\
\hline \multirow{3}{*}{ Target Visibility } & LC & $F(2,118)=20.30, p<0.001$ \\
\hline & LL & $F(4,218)=21.42, p<0.001$ \\
\hline & LC x LL & $F(8,236)=1.96, p=0.053$ \\
\hline \multirow{3}{*}{ Intersection Visibility } & $\mathrm{LC}$ & $F(2,118)=14.12, p<0.001$ \\
\hline & LL & $F(4,218)=77.95, p<0.001$ \\
\hline & LC x LL & $F(8,236)=1.96, p=0.052$ \\
\hline Glare & LL & $\mathrm{F}(4,220)=14.84, \mathrm{p}<0.001$ \\
\hline
\end{tabular}

\section{Pedestrian Visibility}

Subjective ratings of pedestrian visibility were higher for the Approach and Both vs. the Box lighting configuration (FIGURE 8). The difference between age groups approached significance, with younger participants $(M=3.84, S D=1.13)$ providing higher composite scores than older participants $(M=3.14$, $S D=1.36)$. 


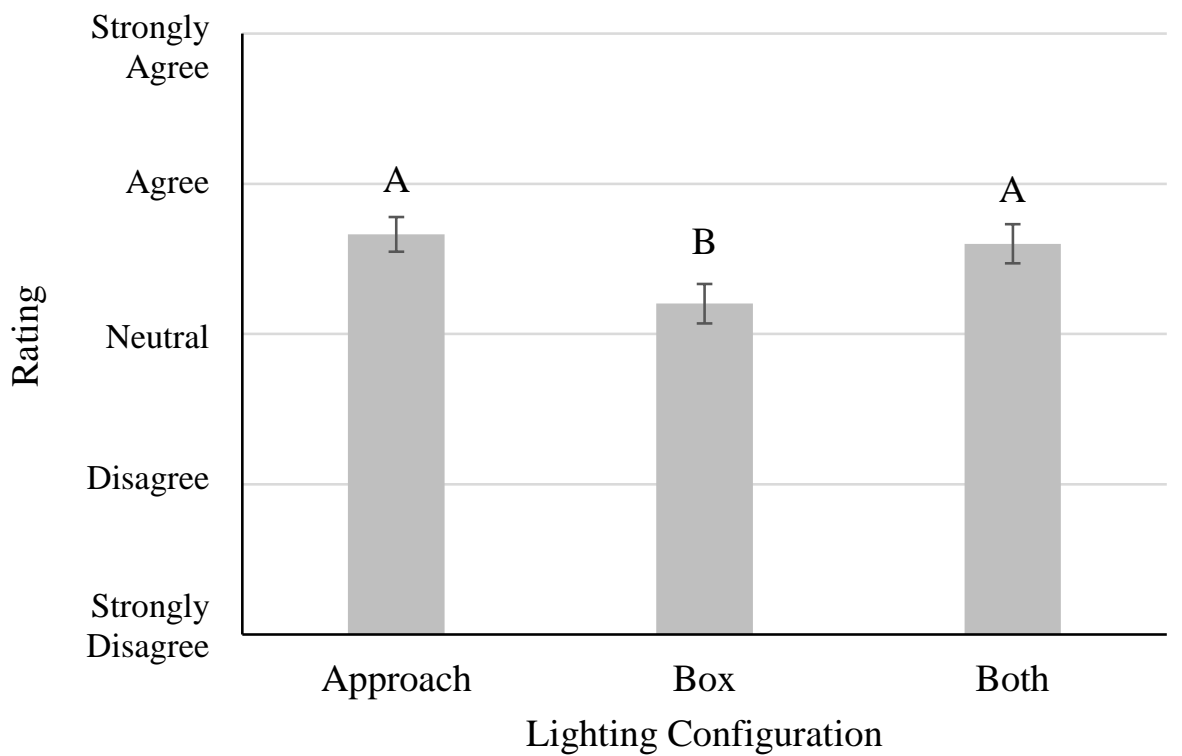

FIGURE 8 Mean ratings of pedestrian visibility in the three lighting configurations. Error bars represent standard errors. Upper case letters indicate groupings based on paired comparisons between lighting configurations.

Analysis of the age $\mathrm{x}$ light level interaction revealed no significant differences between age groups at any of the light levels. A pattern was evident, however, in which younger participants gave higher ratings at all light levels greater than zero (FIGURE 9). Within each age group, increases in light levels were associated with higher ratings. For both groups, ratings appeared to increase for every increment in light level (albeit not significantly). For younger participants, mean Likert-scale composite score exceeded the "neutral" anchor (or a value of 3) at the 8 lux light level, while for the older participants this occurred at 12 lux (FIGURE 9).

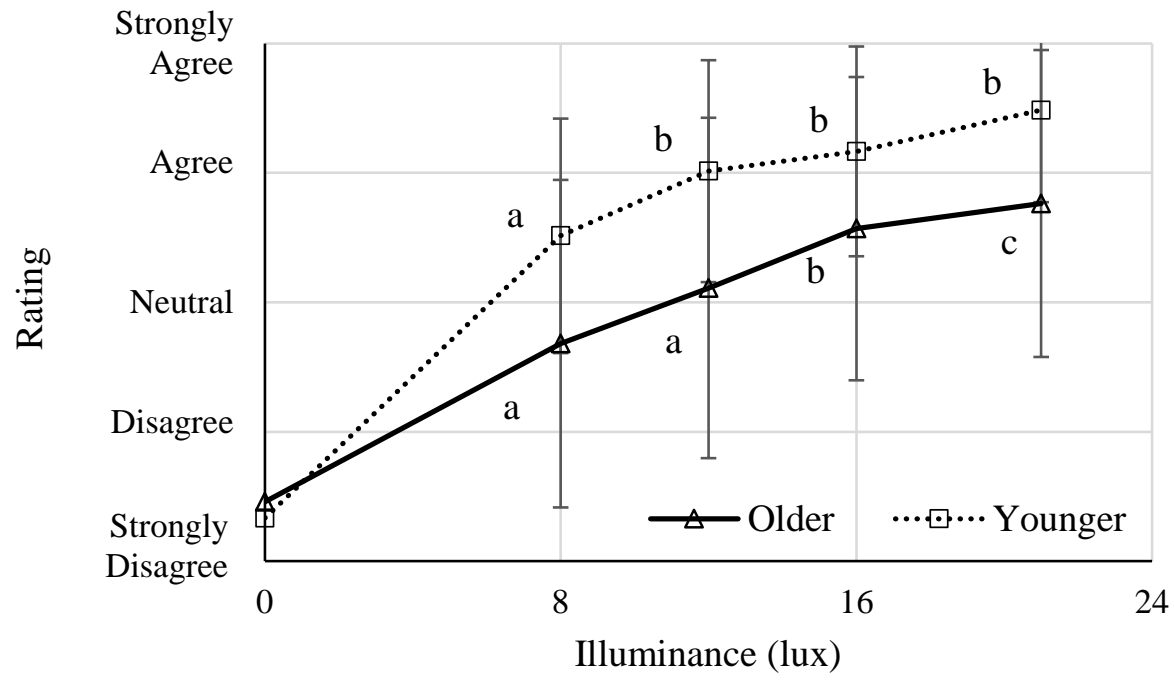

FIGURE 9 Mean ratings of pedestrian visibility at each light level for the two age groups. Error bars represent standard errors. Lower case letters indicate groupings based on paired comparisons between light levels, within each age group.

19 Subjective ratings of target visibility were significantly higher for the Both $(M=3.31, S D=1.06)$ and 
Box $(M=3.7, S D=1.08)$ vs. the Approach $(M=2.6, S D=1)$ lighting configurations. Ratings in the Box configuration were significantly higher than in the Approach configuration at every light level (FIGURE 10). Differences between the Approach and Both lighting configurations, however, were inconsistent and dependent on light level. In each lighting configuration, increases in light level were generally associated with increased target visibility ratings, though only a few paired differences were statistically significant. Plateaus with increasing illuminance were evident only in the Box and Both lighting configurations, for which the respective plateaus were attained at the 8 and 12 lux light levels, respectively. For the Box and Both lighting configurations, mean Likert-scale composite score exceeded the "neutral" anchor (or a value of 3) at the 8 lux light level, whereas it was never exceeded for the Approach lighting configuration (FIGURE 10).

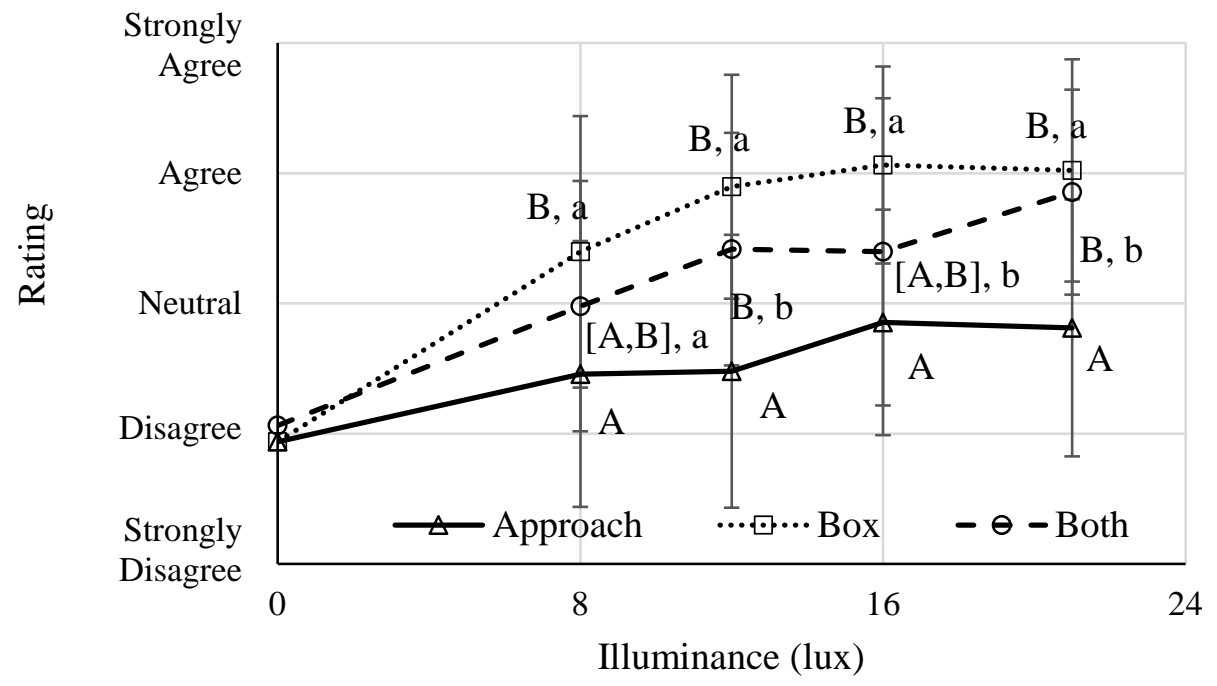

FIGURE 10 Mean ratings of target visibility for each combination of light level and lighting configurations. Error bars represent standard errors. Upper case letters indicate groupings based on paired comparisons between lighting configurations at each light level $>0$. Lower case letters indicate groupings based on paired comparisons between light levels $>0$ within each configuration.

Association between Detection Distance and Perceived Visibility for Targets

Associations between target detection distance and composite ratings of target visibility depended on the lighting configuration. The Box $\left(r_{(24)}=0.43, p=0.03\right)$ and Both $\left(r_{(24)}=0.50, p=0.01\right)$ lighting configurations exhibited significant positive correlations between detection distance and composite ratings of target visibility. This correlation was not significant, though, in the Approach lighting configuration $\left(r_{(24)}=0.02 ; p=0.93\right)$.

\section{Intersection Visibility}

Subjective ratings of intersection visibility were significantly higher for the Both $(M=3.64, S D=0.92)$ and Box $(M=3.94, S D=0.87)$ vs. the Approach $(M=3.29, S D=0.87)$ lighting configurations. Differences in the ratings of the Approach and Box lighting configuration were significant at every light level $>0$, and were highest in the Box lighting configuration (FIGURE 11). In each lighting configuration, increases in light level were associated with increases in ratings of intersection visibility (FIGURE 11), although significant paired differences were found only for the Both configuration. Plateaus were evident in Likert-scale composite scores for all the three lighting configurations. For the Approach and Box lighting configurations, the plateau in subjective ratings of target visibility occurred at the 8 lux light level, while for the Both lighting configuration the plateau occurred at the 12 lux light level. All the three lighting configurations mean Likert composite score exceeded the "neutral" anchor at the 8 lux light level (FIGURE 11). 


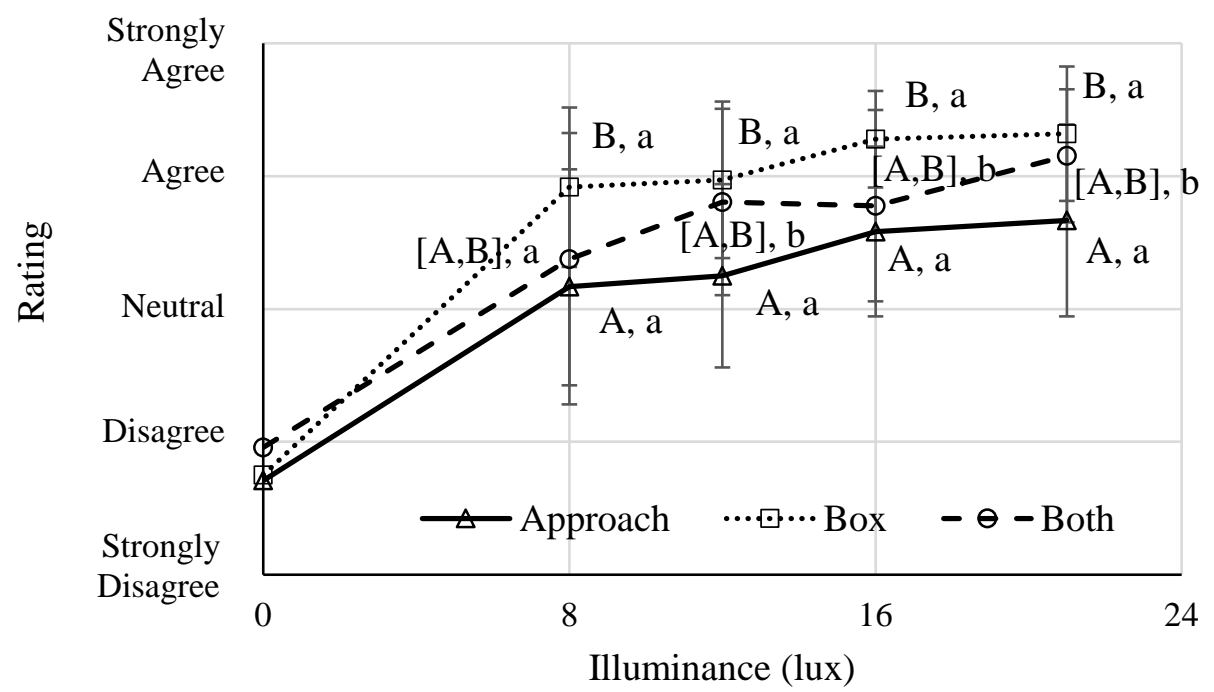

FIGURE 11 Mean ratings of intersection visibility at each light level under all the three lighting configurations. Rrror bars represent standard errors. Upper case letters indicate groupings based on paired comparisons between lighting configurations at each light level $>0$. Lower case letters indicate groupings based on paired comparisons between light levels $>0$.

\section{Glare}

There was a significant main effect of light level on glare, with the ratings at the 0 light level $(M=1.22$, $S D=0.39$ ) having the lowest ratings vs. all other light levels (8 lux: $M=2.28, S D=0.91 ; 12$ lux: $M=$

$102.30, S D=0.86$; 16 lux: $M=2.25, S D=0.81$; and 21 lux: $M=2.13, S D=0.69$ ). However, there was also 11 a significant lighting configuration $\mathrm{x}$ light level interaction, with evidence of a differential influence of light level in the three configurations (Figure 5). Glare was reported to be lowest in the Box configuration and higher in the Approach configuration, for all light levels $>0$, though no pairwise differences were significant. There were also no significant pairwise differences between light levels $(>0)$ within the Approach and Both lighting configurations. For the Box lighting configuration, there were no significant differences between any of the light levels including the 0 . Mean Likert-scale composite scores in all three lighting configurations were less than the "neutral” anchor (FIGURE 12).

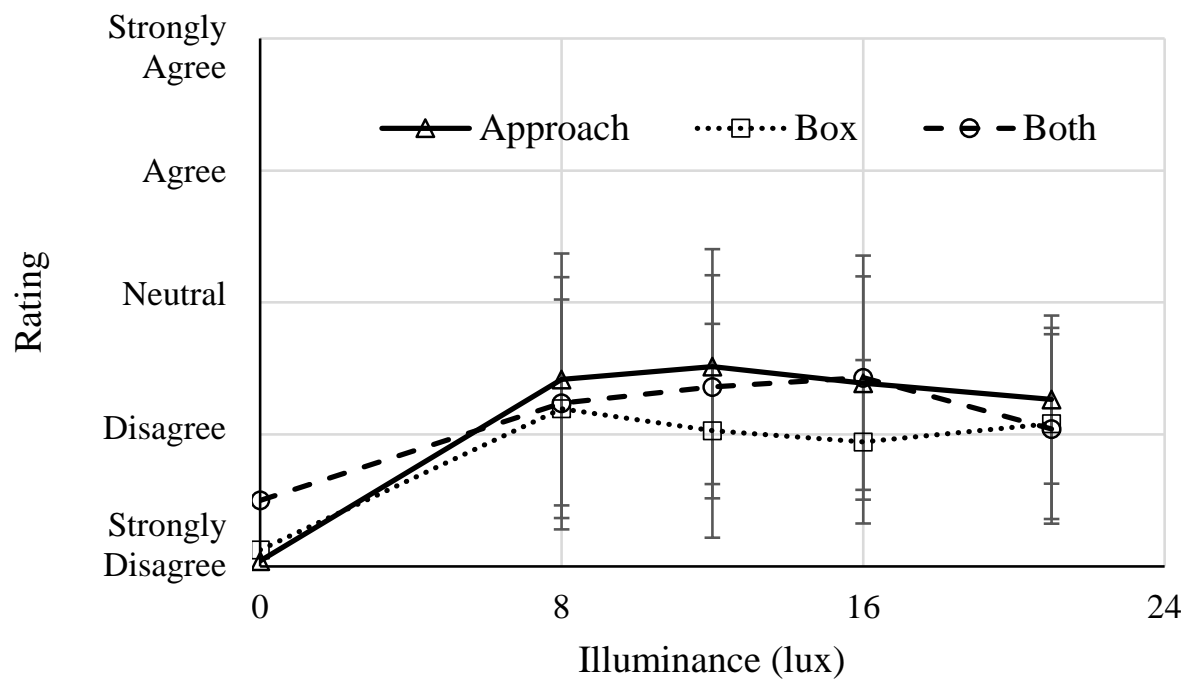

FIGURE 12 Mean ratings of glare at each light level under all the three lighting configurations. Error bars represent standard errors. Note that lower values indicate lower perceived glare. 
1

\section{DISCUSSION}

A primary goal of this study was to assess the effects of intersection lighting configuration and light levels on perceived visibility and glare. Three major findings were evident. First, there were differences in perceived visibility between the three lighting configurations, and the magnitude of these differences depended on the lighting configuration and object size. Second, increases in light level resulted in increased ratings of visibility at some lighting configurations, and there was some evidence of plateaus in these rating with increasing light levels. Third, none of the three lighting configurations were a major source of glare, even at the highest light level.

Perceived visibility depended on the intersection lighting configuration and assessment area. For instance, pedestrian visibility had higher ratings in the Approach lighting configuration, whereas target and intersection visibility were rated higher in the Box lighting configuration. This lack of agreement in the ratings between pedestrian and target visibility may be attributed to the size object involved in each of these assessments. Regarding the former aspect, the pedestrian used here was substantially larger than the targets, and earlier results indicate that larger objects are perceived as being more visible, compared to smaller objects (12).

Regarding perceived target and intersection visibility, the Box lighting configuration was rated higher than the other two lighting configurations at every light level. These results are consistent with our earlier results regarding the visual performance experiment, as assessed by target detection distances (9). Specifically, the Box lighting configuration had longer detection distances at every light level and fewer number of missed target detections, compared to the other two lighting configurations. In the Box and Both lighting configurations, target detection distance and perceived target visibility were positively correlated. In Bhagavathula et al.(9), the longer detection distances in the Box lighting configuration was attributed to the contrast in which the targets were rendered. This conclusion, along with the results regarding perceived target visibility, suggests that the Box lighting configuration renders the targets in adequate contrast (both positive and negative, since target visibility was aggregately assessed for all target locations), resulting in both longer detection distances and higher perceived visibility. Since both perceived visibility and target detection distances in the Both lighting configuration were lower than in the Box lighting configuration, we believe this may have resulted because targets in this lighting configuration were rendered in lower contrast. However, a photometric analysis is required to substantiate the specific contrast levels, which is beyond the scope of this study. In the Approach lighting configuration, all targets were rendered in positive contrast, which may account for the absence of a correlation between target detection distance and perceived visibility in this configuration. Further, positive contrast may also have a differential effect on perceived visibility, depending on the size of the object, since the simulated pedestrian was perceived as more visible than the targets, however, a photometric analysis is required to ascertain this assumption.

The higher ratings of target and intersection visibility in the Box configuration could be attributed to the distribution pattern of the Type V luminaire used to illuminate the intersection box. This pattern is more circular and more uniformly illuminates the intersection compared to the Type II luminaires. The latter, used for illuminating the approach, has a more oval light distribution pattern, and which does not illuminate all the approaches of the intersection (FIGURE 13). This difference could also explain the results in the Both lighting configuration, which had the second highest ratings of both target and intersection visibility, since it included a combination of type II and type V luminaires. Such a combination increased the area illuminated around the intersection, which could have led to participants receiving more visual information from the surroundings, and in turn resulting in higher ratings of target and intersection visibility compared to the Approach lighting configuration. 


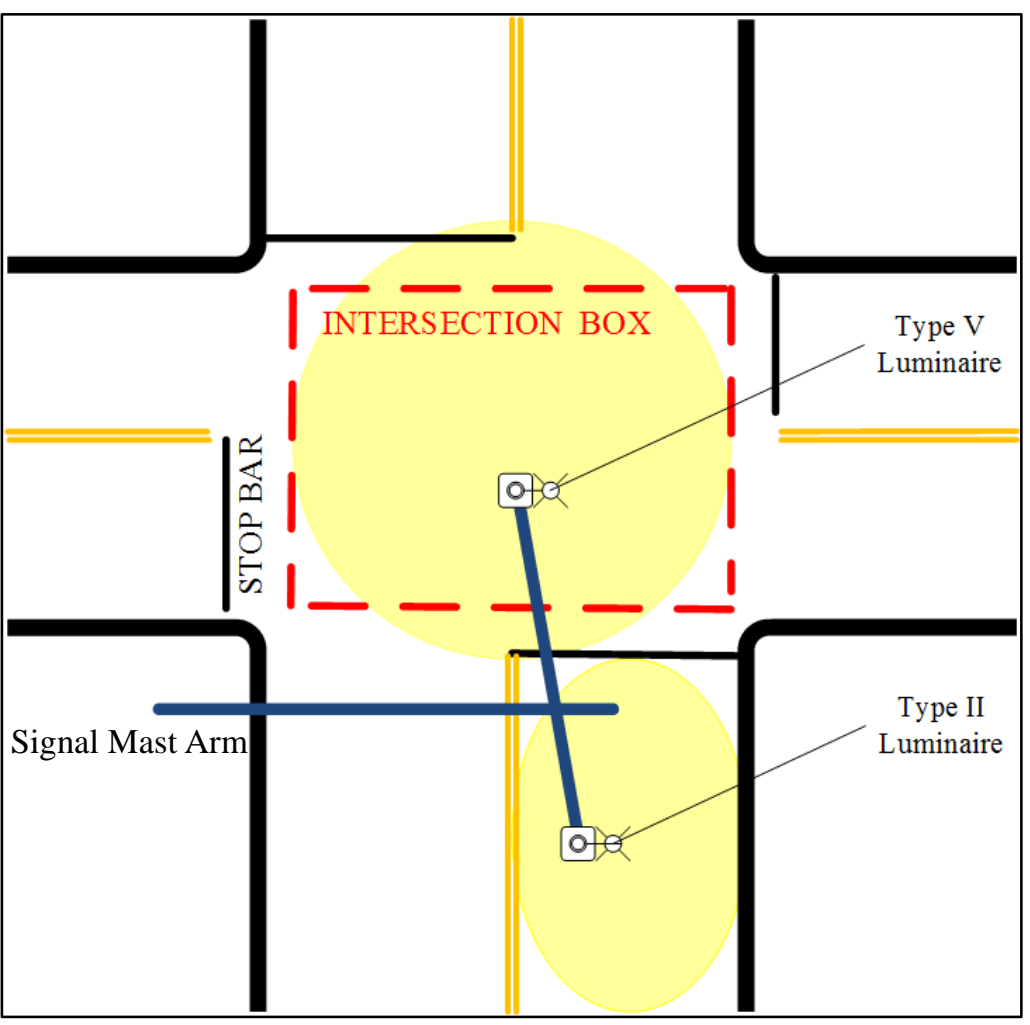

FIGURE 13 Distributions of type V and type II luminaires used to illuminate the intersection box and approach, respectively. The distribution of light (denoted by the yellow color) is not photometrically accurate and is only included to represent light distributions by the luminaires for illustrative purposes.

Ratings of pedestrian visibility exceed the "neutral" anchor $(>3)$ in all three lighting configurations, albeit at different light levels. For the Approach lighting configuration, this occurred at 8 lux, whereas for the Both and Box lighting configurations this threshold was crossed at 12 lux. These pedestrian rating results align with results of Minoshima et al. (17), which indicated that an average surface illuminance of 10 lux or higher yielded mean rating higher than 3 regardless of luminaire layout or lighting configuration. However, results here regarding perceptions here of intersection visibility and target visibility did not completely align with those of Minoshima et al. (17). Mean ratings of intersection visibility here exceeded the "neutral" anchor for all three lighting configurations at an light level lower (8 vs. 10 lux) than what was reported by Minoshima et al (17). For target visibility, mean ratings in the Approach lighting configuration never exceeded the "neutral" anchor, even at the highest light level studied (21 lux), whereas in the Box and Both lighting configurations it was exceeded at 8 and 12 lux, respectively. These results indicate that the lighting configuration (or the part of the intersection that is illuminated) plays an important role in influencing perceived intersection and target visibility, but less of a role in influencing pedestrian visibility.

Increases in light level generally resulted in higher ratings of pedestrian, target, and intersection visibility in all the three lighting configurations. These results are similar to those of Minoshima et al. (17) and Janoff (12), who found higher subjective ratings of visibility with increases in light level. Assessments of the effects of light levels within each lighting configuration showed that some plateaus occurred, but such patterns depended on the specific lighting configuration and perceptual measure. For the Box and Both lighting configurations this occurred at 8 and 12 lux light levels, whilst the Approach lighting configuration exhibited a plateau for intersection visibility at the 8 lux light level.

The Box lighting configuration led to the lowest glare ratings amongst the three lighting configurations, though none of the three intersection lighting configurations appeared to be a major 
source of glare. This result in not in agreement with existing research and the discrepancy could be attributed to different experimental methodologies used to assess perceived ratings of glare. In this study, the only source of glare were the luminaires used to illuminate the intersection whereas in the other studies the sources of glare were primarily headlamps or simulated headlamps of vehicles which direct substantially more light into driver's eyes. Further, detailed glare assessments as to the specific effects of fixture, background luminance etc. would require additional measurements and analyses which were not conducted as a part of this experiment.

Individual differences also did not appear to be a major source of variability. There were no significant differences found between males and females for any of the rating, and also no significant differences between the two age groups examined except for participant visibility. Regarding the latter, younger participants reported higher levels of pedestrian visibility for all non-zero light levels.

This study has some limitations that should be noted. For example, the experimental setting was somewhat limited in that there was no additional traffic and the signal lights were turned off at the intersection, and subjective ratings (including glare) were evaluated at single point on the approach to the intersection. These conditions were used to minimize potential confounding effects of glare and signal phase timing that could have affected drivers' perceptions of visibility and glare. Taking subjective ratings at multiple points could have prolonged the experimental duration and fatigued the participants, which could have potentially confounded the results. Further, the current results may be most valid for isolated/rural intersections, which are illuminated by a single light source and for which none of the approaching roads have continuous lighting. On roads with continuous lighting, additional light from luminaires might substantially affect contrast and subsequently the visibility of objects located at the intersection. Future work on intersection lighting should consider the effects of the presence of multiple vehicles, signal phase, and continuous lighting to more accurately understand the effects of intersection lighting design on visibility and glare.

\section{CONCLUSIONS}

Illuminating different parts of an intersection leads to important differences in perceived visibility and glare, and the patterns of these differences are generally consistent with measures of visual performance (9). Illuminating the intersection box yielded the highest ratings of target and intersection visibility and lowest ratings of glare. Even though the Approach lighting configuration had higher ratings of pedestrian visibility than the Box lighting configuration, target visibility ratings in this configuration were lower than the "neutral" anchor at every light level. Moreover, at light levels greater than 8 lux, pedestrian visibility ratings in all three lighting configurations exceeded the "neutral" anchor and did not differ significantly from one another. For the Box lighting configuration, plateaus in perceived visibility differed between assessment areas, occurring at light levels of 8 lux (or 7 lux mean intersection illuminance for target and intersection visibility) or 12 lux (or 10 lux mean intersection illuminance for pedestrian visibility). Ratings of the Approach lighting configuration were less consistent, yielding the highest ratings for pedestrian visibility and the lowest ratings of target and intersection visibility. The Box lighting configuration also had lowest ratings of glare, although none of the three lighting configurations was a major source of glare. Additionally, the Box lighting configuration required only one luminaire to illuminate the entire intersection, whereas the Approach and Both lighting configurations require at a minimum, as many luminaires as there are approaches (to illuminate all pedestrian-vehicle conflict locations), making these latter configurations more expensive. These results can have important implications for lighting design of rural/isolated intersections illuminated by a single light source. Specifically, they suggest that illuminating the intersection box is likely to have multiple parallel benefits, with longer detection distances, higher perceived visibility, and lower glare.

\section{ACKNOWLEDGMENTS}

The authors of this report would like to acknowledge the support of the stakeholders of the National Surface Transportation Safety Center for Excellence (NSTSCE): Tom Dingus from the Virginia Tech Transportation Institute, John Capp from General Motors Corporation, Chris Hayes from Travelers Insurance, Martin Walker from the Federal Motor Carrier Safety Administration, and Cathy McGhee 
from the Virginia Department of Transportation and the Virginia Center for Transportation Innovation and Research. The NSTSCE stakeholders have jointly funded this research for the purpose of developing and disseminating advanced transportation safety techniques and innovations.

\section{AUTHOR CONTRIBUTION STATEMENT}

The authors confirm contribution to the paper as follows: study conception and design: Rajaram Bhagavathula, Ronald B. Gibbons; data collection: Rajaram Bhagavathula; analysis and interpretation of results: Rajaram Bhagavathula, Ronald B. Gibbons, Maury A. Nussbaum; draft manuscript preparation: Rajaram Bhagavathula, Maury A. Nussbaum. All authors reviewed the results and approved the final version of the manuscript.

\section{REFERENCES}

1. NHTSA, Traffic Safety Facts 2013. 2014, National Highway Traffic Safety Administration: Washington, DC.

2. Wortman, R. and M. Lipinski, RURAL AT-GRADE INTERSECTION ILLUMINATION. 1974, University of Illinois ar Urbana-Champaign.

3. Isebrands, H.N., et al., Safety Impacts of Street Lighting at Isolated Rural Intersections - Part II. 2006, Iowa State University, Ames, Minnesota Department of Transportation. p. 120.

4. $\quad$ Smadi, O., N. Hawkins, and B. Aldemir-Bektas, Roadway Lighting and Safety: Pase II Monitoring Quality, Durability and Efficiency. 2011, Center for Transportation Research and Education.

5. Bullough, J., E. Donnell, and M. Rea, To illuminate or not to illuminate: Roadway lighting as it affects traffic safety at intersections. Accident Analysis \& Prevention, 2013. 53(0): p. 65-77.

6. Donnell, E.T., R.J. Porter, and V.N. Shankar, A framework for estimating the safety effects of roadway lighting at intersections. Safety Science, 2010. 48(10): p. 1436-1444.

7. Bhagavathula, R., R.B. Gibbons, and C.J. Edwards, Relationship between roadway illuminance level and nighttime rural intersection safety. Transportation Research Record: Journal of the Transportation Research Board, 2015(2485): p. 8-15.

8. $\quad$ Edwards, C., Lighting Levels for Isolated Intersections Leading to Safety Improvements. 2015, University of Minnesota.

9. Bhagavathula, R., R.B. Gibbons, and M.A. Nussbaum, Effects of Intersection Lighting Design on Nighttime Visual Performance of Drivers. LEUKOS, 2018. 14(1): p. 25-43.

10. Gallagher, V., B. Koth, and M. Freedman, The specification of street lighting needs. 1975.

11. Janoff, M.S., et al., EFFECTIVENESS OF HIGHWAY ARTERIAL LIGHTING FINAL REPORT. 1977.

12. Janoff, M., Subjective ratings of visibility and alternative measures of roadway lighting. Journal of the Illuminating Engineering Society, 1989. 18(1): p. 16-28.

13. Alferdinck, J. and J. Varkevisser, Discomfort Glare From D1 Headlamps of Different Size, Report IZF 1991 C-21. Soesterberg, Netherlands: TNO Institute for Perception, 1991.

14. Schmidt-Clausen, H.-J. and J.T.H. Bindels, Assessment of discomfort glare in motor vehicle lighting. Lighting Research and Technology, 1974. 6(2): p. 79-88.

15. Sivak, M., C.J. Simmons, and M. Flannagan, Effect of headlamp area on discomfort glare. Lighting Research and Technology, 1990. 22(1): p. 49-52.

16. Theeuwes, J., J.W. Alferdinck, and M. Perel, Relation between glare and driving performance. Human Factors: The Journal of the Human Factors and Ergonomics Society, 2002. 44(1): p. 95107.

17. Minoshima, O., et al. Research on Accident Reduction by Intersection Lighting. in Road Engineering Association of Asia and Australasia (REAAA) Conference. 2006. Philippines.

18. Mayeur, A., R. Bremond, and J.M.C. Bastien, The effect of the driving activity on target detection as a function of the visibility level: Implications for road lighting. Transportation Research Part F: Traffic Psychology and Behaviour, 2010. 13(2): p. 115-128. 
19. Janoff, M.S., The Relationship between Small Target Visibility and a Dynamic Measure of Driver Visual Performance. Journal of the Illuminating Engineering Society, 1993. 22(1): p. 104-112.

20. Bhagavathula, R. and R.B. Gibbons. Role of Expectancy, Motion and Overhead Lighting on Nighttime Visibility. in Proceedings of the Human Factors and Ergonomics Society Annual Meeting. 2013. SAGE Publications.

21. Bhagavathula, R., R.B. Gibbons, and C.J. Edwards. Effect of Static and Moving Objects on Driver Eye Movements and Detection Distances. in Transportation Research Board 91st Annual Meeting. 2012.

22. Gibbons, R.B., et al., Development of visual model for exploring relationship between nighttime driving behavior and roadway visibility features. Transportation Research Record: Journal of the Transportation Research Board, 2012. 2298(1): p. 96-103.

23. Gibbons, R., et al., Evaluation of Impact of Modern Headlamp Technology on Design Criteria for Sag Vertical Curves. Transportation Research Record: Journal of the Transportation Research Board, 2013(2384): p. 65-73.

24. Janoff, M., The effect of headlights on small target visibility. Journal of the Illuminating Engineering Society, 1992. 21(2): p. 46-53.

25. IES, RP-8-14 Roadway Lighting. 2014, Illuminating Engineering Society of North America.

18 26. AASHTO, A policy on geometric design of highways and streets: 2011. Vol. 6th. 2011,

27. Nunnally, J.C. and I.H. Bernstein, Psychometric theory. 1994, New York: McGraw-Hill. 